\title{
Dynamics of rough interfaces in Chemical Vapor Deposition: experiments and model for silica films
}

\author{
Fernando Ojeda ${ }^{1}$, Rodolfo Cuerno ${ }^{2}$, Roberto Salvarezza $^{3}$, and Luis Vázquez ${ }^{1}$ \\ ${ }^{1}$ Instituto de Ciencia de Materiales de Madrid (CSIC), Cantoblanco, E-28049 Madrid, SPAIN \\ 2 Departamento de Matemáticas and Grupo Interdisciplinar de Sistemas Complicados, \\ Universidad Carlos III de Madrid, Avenida de la Universidad 30, E-28911 Leganés, SPAIN \\ 3 INIFTA, Sucursal 4, Casilla de Correo 16, (1900) La Plata, ARGENTINA
}

(February 1, 2008)

\begin{abstract}
We study the surface dynamics of silica films grown by low pressure chemical vapor deposition. Atomic force microscopy measurements show that the surface reaches a scale invariant stationary state compatible with the Kardar-Parisi-Zhang (KPZ) equation in three dimensions. At intermediate times the surface undergoes an unstable transient due to shadowing effects. By varying growth conditions and using spectroscopic techniques, we determine the physical origin of KPZ scaling to be a low value of the surface sticking probability, related to the surface concentration of reactive groups. We propose a stochastic equation that describes the qualitative behavior of our experimental system.
\end{abstract}

PACS number(s): 68.55.Jk, 64.60.Ht, 81.15.Gh, 05.10.Gg

The dynamics of growing interfaces has become of increasing interest in order to understand the physical processes that determine film quality [1]. In the absence of morphological instabilities, many surfaces evolving out of equilibrium display time and space scale invariance, i.e. such surfaces are rough. A successful framework for the study of rough interface dynamics has been the formulation of stochastic differential equations for the surface height $h(\boldsymbol{r}, t)$, where $\boldsymbol{r}$ denotes a site of a two-dimensional substrate, and $t$ is time. A prominent example is the Kardar-Parisi-Zhang (KPZ) equation [2]

$$
\partial_{t} h=\nu \nabla^{2} h+(\lambda / 2)(\nabla h)^{2}+\eta(\boldsymbol{r}, t)
$$

expected to describe the large length scale dynamics of any rough surface growing in the absence of specific conservation laws. In Eq. (11), $\nu$ and $\lambda$ are constants. The first term on the rhs is sometimes called a surface tension term, as it tends to smooth the interface through evaporation-condensation processes [1]. The nonlinear term accounts for growth along the local normal direction (lateral growth), and $\eta(\boldsymbol{r}, t)$ is a Gaussian white noise of constant strength $2 D$, accounting for microscopic fluctuations, e.g., in a deposition beam.

One of the techniques for thin film production which might be expected to lead to surfaces described by (1) is Chemical Vapor Deposition (CVD). CVD is very widely used for technological applications and industrial devices [3]. It is also very interesting from the fundamental point of view due to its conceptual similarities with other growth techniques such as electrodeposition. Thus, a considerable effort has been made to model CVD growth [1 6] using partial differential equations. These models predict the development of unstable morphologies within the time ranges studied, contradicting the above expectation of KPZ scale invariant behavior. Let us note that, to date, very few experimental systems are known whose scaling is described by KPZ in three dimensions [7]. Moreover, on the fundamental level there are no experiments addressing the long time behavior of the surface morphologies produced by CVD, and the identification of the determining physico-chemical mechanisms.

In this Letter we study the interface dynamics of low pressure $\mathrm{SiO}_{2}$ CVD films by Atomic Force Microscopy (AFM), Raman and infrared spectroscopies, and numerical simulations. $\mathrm{SiO}_{2}$ films were studied because of their amorphous nature, to avoid Schwoebel barrier effects on the surface dynamics [8], and to prevent formation of facets that can also alter the scaling behavior [6]. Our experimental system displays a transient unstable behavior related to the gas phase transport character of CVD growth [4], but a stationary state is achieved which, rather, is compatible with KPZ scaling. The surface dynamics is well described, over a wide spatial and temporal range (up to 2 days of deposition time), by a stochastic differential equation in which surface diffusion, shadowing effects, and lateral growth are allowed for.

Amorphous $\mathrm{SiO}_{2}$ films were grown at a temperature $T$ $=723 \mathrm{~K}$ on $\mathrm{Si}(100)$ substrates in a hot wall, horizontal, low pressure tubular CVD reactor. The precursor gases were silane (diluted at $2 \%$ in nitrogen, $99.999 \%$ purity) and oxygen (99.9992\% purity), with an oxygen/silane ratio equal to 20 and a total gas flow rate of $50 \mathrm{sccm}$. The chamber pressure was 1.4 Torr. The film thickness increases linearly with deposition time at a constant growth rate of $20 \mathrm{~nm} / \mathrm{min}$. Films were deposited in the range 5 $\min \leq t \leq 2$ days. The surface morphology was characterized by AFM (Nanoscope III from Digital Instruments, CA) operating in tapping mode at ambient conditions up to a scale of $50 \mu \mathrm{m}$ using silicon cantilevers.

AFM imaging of our silica films shows (Fig. 1) a de- 
posit formed initially by small rounded grains $30-60 \mathrm{~nm}$ in size. A similar morphology has been reported on amorphous silicon films deposited by thermal evaporation [9]. As deposition proceeds, structures resembling mountains and valleys appear at larger length scales increasing in size, until a stationary regime is attained in the 15-30 hours range.

Power Spectral Density (PSD) (or surface power spectrum) plots of the surface morphology at different times are displayed in Fig. 2. For $t<35 \mathrm{~min}$ and small enough wave vector $k$, the PSD follows that of the initial substrate, while for $t>35 \mathrm{~min}$ and $k<k_{c}$ it takes a constant $k$-independent value, interface portions separated by distances $r>1 / k_{c}$ being uncorrelated. For a rough interface [1], the value of $k_{c}$ decreases with time as $k_{c} \sim t^{-1 / z}$, where $z$ is the dynamic exponent. Indeed, from the log$\log$ plot of $k_{c}$ vs $t$ (Fig. 3), a slope value $z=1.6 \pm 0.1$ is obtained. Therefore, the time behavior of our CVD growth process is well described by that of a self-affine rough surface. The same conclusion is obtained from the spatial behavior of the surface morphology. Specifically, for a two-dimensional rough interface the PSD behaves as [1] $\operatorname{PSD}(k) \sim 1 / k^{2 \alpha+2}$, with $\alpha$ the roughness exponent. In Fig. 2, as time proceeds, up to three spatial regions can be observed:

i) For $t<50 \mathrm{~min}$ we can distinguish two regions: Region I, for $k>k_{0}$, features $\alpha_{I}=0.99 \pm 0.04 ; k_{0}$ initially decreases from $0.04 \mathrm{~nm}^{-1}$ for $t=20 \mathrm{~min}$ to a constant value of $0.02 \mathrm{~nm}^{-1}$ for $t \geq 50 \mathrm{~min}$. Region II with $\alpha_{I I}=0.75 \pm 0.03$ is observed for $k_{1}<k<k_{0}$, where $k_{1}$ changes from $0.025 \mathrm{~nm}^{-1}$ for $t=20 \min$ to $0.0034 \mathrm{~nm}^{-1}$ for $t=50 \mathrm{~min}$.

ii) For $t>50 \mathrm{~min}$ a new region III appears for $k_{c}<k<k_{1}$, with $\alpha_{I I I}=0.42 \pm 0.03$; as noted above, $k_{c}$ decreases with time. Regions I and II are still observed.

The above behavior of the PSD is compatible with that of the surface width, $\left.W^{2}(t)=\overline{\left\langle(h(\boldsymbol{r}, t)-\bar{h}(t))^{2}\right.}\right\rangle$, which we measure independently (Fig. 3). The bar denotes spatial average. For a rough interface, initially $W(t) \sim t^{\alpha / z}=t^{\beta}$, as long as $t \ll L^{z}$, with $L$ a measure of the spatial extent of the system. For $t \gg L^{z}$, the interface saturates into a stationary state for which $W \sim L^{\alpha}$. As a difference with the PSD, we observe only two temporal behaviors in $W(t)$, corresponding to regions II and III of the PSD. For $t<50 \mathrm{~min}$, the width is described by the growth exponent $\beta_{I I}=0.42 \pm 0.04$, while for 50 $\min \leq t \leq 15$-30 hours, $W(t)$ data are consistent with $\beta_{I I I}=\alpha_{I I I} / z=0.26 \pm 0.03$ as obtained from the PSD data. For $t>15-30$ hours, the width $W(t)$ saturates to a constant value. Note that the contribution of the short length scales (region I) to the surface width is masked by the spatial region II. However, despite the few data points for $t \leq 50 \mathrm{~min}$, an attempt to estimate $\beta_{I}$ from the PSD curves [10] leads to $\beta_{I}=0.28 \pm 0.09$. Regarding the long distance properties of our system, the observed
$\alpha_{I I I}=0.42 \pm 0.03, z=1.6 \pm 0.1$, and $\beta_{I I I}=0.26 \pm 0.03$ are close, within experimental errors, to the approximate exponents for the KPZ universality class in three dimensions, namely [1], $\alpha_{K P Z}=0.39, z_{K P Z}=1.63$, and $\beta_{K P Z}=0.24$. Thus, the asymptotic scaling behavior of the growing CVD surface is of the KPZ class, an experimental result very rarely found in three dimensions [7].

It is important to understand the origin of the KPZ regime under our experimental conditions. It is known 11] that, for CVD systems with a low surface reaction kinetics (low sticking probability $s$ ), lateral growth, which is the fingerprint of KPZ behavior, is promoted leading to the so-called conformal growth. The mechanism inducing conformal growth is the reemission of the precursor (i.e., depositing) species due to a low sticking probability. For our experimental conditions $s$ has actually been reported 12 to decrease with temperature from $s=0.5$ at $573 \mathrm{~K}$ down to $s=0.08$ at $723 \mathrm{~K}$. Also, the growth conformality of submicron trenches by $\mathrm{SiO}_{2}$ films has been reported [12,13] to improve as $T$ increases. Physically, $s$ can be related to the concentration of reactive sites at the growing film surface, which for our system are mainly associated with hydrogenated groups, such as $\mathrm{Si}-\mathrm{OH}$ and $\mathrm{Si}-\mathrm{H}$, and with strained siloxane (Si-O-Si) groups, rather than with the less reactive relaxed siloxane groups [14].

To study the role played by the sticking probability on our film morphology, we have grown $\mathrm{SiO}_{2}$ films at lower $T=611 \mathrm{~K}$ (higher $s$ ) with the same growth rate $(\approx 20 \mathrm{~nm} / \mathrm{min})$. Analysis of our films by Raman and infrared spectroscopies shows [15] that the $611 \mathrm{~K}$ films indeed present a significantly higher concentration of reactive groups than the $723 \mathrm{~K}$ films, in agreement with the higher sticking probability expected for the low temperature growth. Moreover, regarding the surface dynamics of the low $T$ films, neither the KPZ nor the saturation regimes were found after 2 days of deposition. In Fig. 4 we plot the PSD of a film grown at $611 \mathrm{~K}$ after 2 days. The KPZ region does not appear but only regions I and II do. In Fig. 4 (inset) we plot $W(t)$ for the same set of films. Again, only unstable growth is obtained for long times. From the point of view of Eq. (11), these experiments suggest that when $T$ is reduced the effective $\lambda$ coefficient of the KPZ term decreases. These results are analogous to the crossover found between diffusionlimited aggregation and Eden growth when tuning the sticking probability 16, and allow us to identify region II in the scaling behavior at the high tempererature value $(T=723 \mathrm{~K})$ as an unstable transient.

The above results suggest that the KPZ equation might be a good starting point to describe the observed behavior. However, in our system we expect the first term in equation (11) to be negligible, since the $\mathrm{SiO}_{2}$ vapor pressure is extremely low (i.e. $\nu$ is very small) in our temperature range, whereas surface stabilization by surface diffusion seems to be relevant [9]. In fact, $\alpha_{I} \approx 0.99$ and $\beta_{I} \approx 0.28$ are consistent within experimental errors 
with the linear theory of surface diffusion [17]. On the other hand, due to the large values of the effective exponents $\alpha_{I I}$ and $\beta_{I I}$ and in view of the discussion above, we believe region II corresponds to unstable growth. This instability can be related to shadowing effects which occur in CVD 田原] due to the random walk motion of the depositing particles. Nonlinear surface diffusion [18] can be discarded as the origin of the unstable behavior in region II since it leads to anomalous scaling [1], not present in our measurements. Similarly, the unstable equation for $h$ derived in [6] can be ruled out as a description of the unstable behavior in region II because it leads to a non constant growth rate, incompatible with our experimental setup. Thus, we propose the following continuum equation to describe the silica CVD growth:

$$
\partial_{t} h=-K \nabla^{4} h+\varepsilon \theta / \bar{\theta}+(\lambda / 2)(\nabla h)^{2}+\eta(\boldsymbol{r}, t) .
$$

In Eq. (2), $K$ and $\varepsilon$ are positive constants; the first term on the rhs represents relaxation by surface diffusion and the second term represents geometric shadowing effects, $\theta$ being the local exposure angle and $\bar{\theta}$ being the spatial average of $\theta$ [19]. In order to check the validity of (2) we have performed numerical simulations in two dimensions (three dimensional simulations are limited to small system sizes [201). In Fig. 5 we plot the time evolution of the PSD for $K=D=1, \varepsilon=1 / 2$, and two values of the strength of the KPZ non-linearity $\lambda=0.2,3$. For these sets of parameters we have employed system sizes up to $L=8192$ in order to reliably identify the different scaling regimes. For $\lambda=3$ (solid line in Fig. 5), and as experimentally observed at high $T$ (Fig. 2), the surface PSD evolves from a scaling regime dominated by surface diffusion at short distances to KPZ scaling at large length scales, through an unstable transient due to the shadowing effects 21]. Also in agreement with the experimental data, after a certain growth time both $k_{0}$ and $k_{1}$ are frozen, and no anomalous scaling is observed. The time evolution of the surface width is shown in the inset of Fig. 5 for the same parameters. Again, for $\lambda=3$, it resembles quite closely that shown in Fig. 3 [21] since, as $t$ increases, the behavior changes from surface diffusion to unstable growth and finally to KPZ scaling. For smaller $\lambda=0.2$ (dashed lines in Fig. 5), corresponding experimentally to low $T$ (Fig. 4), we observe from the PSD and $W(t)$ behaviors that the crossover from the unstable region II to KPZ behavior takes place, if at all, at longer time and larger length scales. Similar behaviors to those in Fig. 5 are obtained for other parameter sets 15], provided that the relative weight of the different contributions is preserved.

In summary, we have found that low pressure CVD growth of silica films is governed by the relative balance between surface diffusion, shadowing and lateral (KPZ) growth. Our experimental system is well described by a differential continuum stochastic equation in which these three mechanisms are allowed for. Moreover, our study allows to link the value of the effective $\mathrm{KPZ}$ nonlinearity to the physical and chemical properties of the growing interface. Thus, we conclude that the observation of asymptotic KPZ scaling is favoured under growth conditions (e.g., high temperatures) which promote a low sticking probability of the depositing species.

This work has been performed within the CONICETCSIC and Programa de Cooperación con Iberoamérica (MEC) research programs, and has been partially supported by CAM grants 7220-ED/082, 07N-0028, and DGES grants MAT97-0698-C04, PB96-0119. F. O. acknowledges support by CAM.

[1] A.-L. Barabási and H. E. Stanley, Fractal Concepts in Surface Growth (Cambridge University Press, Cambridge, 1995); J. Krug, Adv. Phys. 46, 139 (1997).

[2] M. Kardar, G. Parisi, and Y.-C. Zhang, Phys. Rev. Lett. 56, 889 (1986).

[3] K. F. Jensen and W. Kern in Thin Film Processes II, J. L. Vossen and W. Kern eds. (Academic, Boston, 1991).

[4] C. H. J. Van den Brekel and A. K. Jansen, J. Cryst. Growth 43, 364 (1977).

[5] B. J. Palmer and R. G. Gordon, Thin Solid Films 158, 313 (1988).

[6] G. S. Bales, A. C. Redfield, and A. Zangwill, Phys. Rev. Lett. 62, 776 (1989).

[7] J. Chevrier et al., Europhys. Lett. 16, 737 (1991); E. A. Eklund et al., Phys. Rev. Lett. 67, 1759 (1991); R. Paniago et al., Phys. Rev. B 56, 13442 (1997).

[8] R. L. Schwoebel, J. Appl. Phys. 40, 614 (1968).

[9] H.-N. Yang et al., Phys. Rev. Lett. 76, 3774 (1996).

[10] By integrating the PSD curves from the maximum $k$ available down to $k_{0}$.

[11] M. J. Cooke and G. Harris, J. Vac. Sci. Tech. A 7, 3217 (1989).

[12] K. Watanabe and H. Komiyama, J. Electrochem. Soc., 137, 1222 (1990).

[13] M. Ikegawa and J. Kobayashi, J. Electrochem. Soc. 136, 2982 (1989); D. G. Coronell and K. F. Jensen, ibid. 141, 2545 (1994).

[14] R. K. Iler, The chemistry of silica (J. Wiley \& Sons, New York, 1979); T. A. Michalske and B. C. Bunker, J. Appl. Phys. 56, 2686 (1984); Q. Sun et al., Europhys. Lett. 43, 47 (1998).

[15] F. Ojeda et al., in preparation.

[16] P. Meakin, Fractals, scaling and growth far from equilibrium (Cambridge University Press, Cambridge, 1998).

[17] J. Villain, J. Physique I (France) 1, 19 (1991); Z.-W. Lai and S. Das Sarma, Phys. Rev. Lett. 66, 2348 (1991).

[18] F. Family and J. Amar, Fractals 1, 753 (1993).

[19] Related equations have been studied by R. P. U. Karunasiri, R. Bruinsma, and J. Rudnick, Phys. Rev. Lett. 62, 788 (1989) and J. H. Yao, C. Roland, and H. Guo, Phys. Rev. A 45, 3903 (1992).

[20] J. H. Yao and H. Guo, Phys. Rev. E 47, 1007 (1993).

[21] The values of $\alpha$ and $z$ depend on dimensionality, and thus differ in the experiments and in our numerical simula- 
tions. However, the qualitative behaviors are the same: for Fig. 5 we obtain for the $\lambda=3$ case $\alpha_{I}^{s}=1.5 \pm 0.1$ $\left(\beta_{I}^{s}=0.37 \pm 0.01\right), \alpha_{I I}^{s}=0.86 \pm 0.04\left(\beta_{I I}^{s}=0.45 \pm 0.02\right)$, and $\alpha_{I I I}^{s}=0.5 \pm 0.01\left(\beta_{I I I}^{s}=0.33 \pm 0.02\right)$, consistent, respectively, with linear surface diffusion, unstable growth, and KPZ scaling in two dimensions.

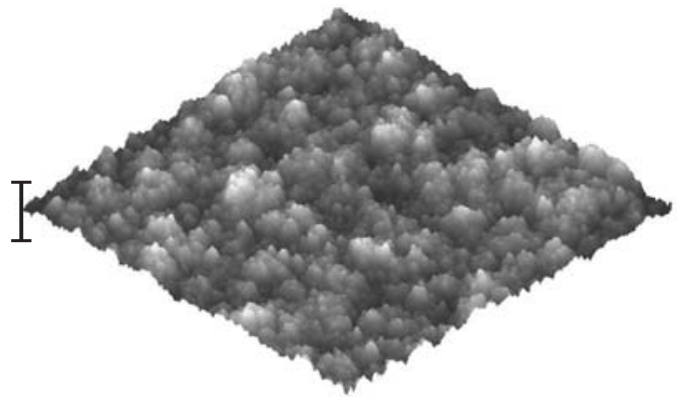

FIG. 1. AFM $3 \times 3 \mu \mathrm{m}^{2}$ image of amorphous $\mathrm{SiO}_{2}$ films deposited at $T=723 \mathrm{~K}$ for $t=75 \mathrm{~min}$. The vertical bar represents $400 \mathrm{~nm}$.

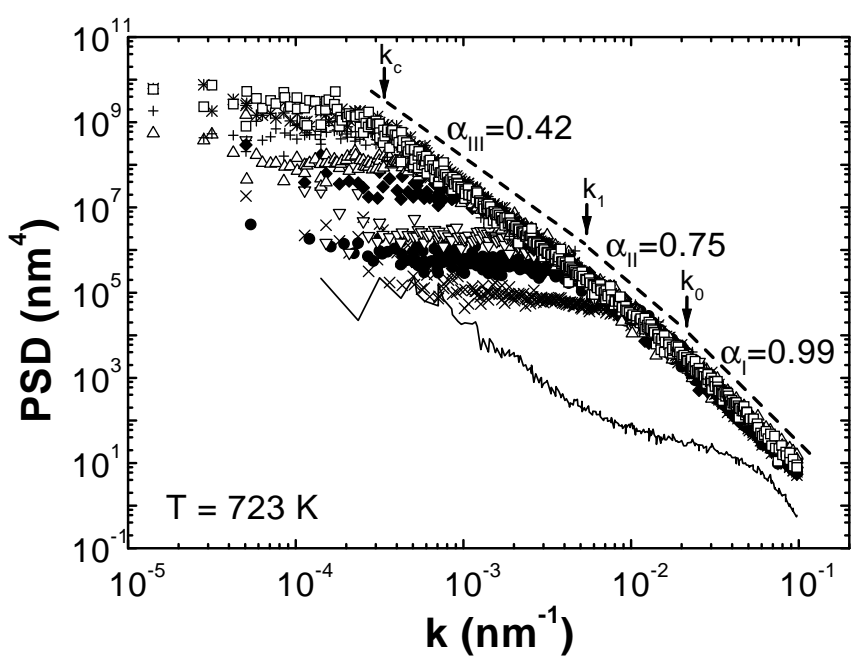

FIG. 2. PSD curves from AFM images of the $\mathrm{SiO}_{2}$ surface at different times: substrate (solid line), $5 \mathrm{~min}(\times), 20 \mathrm{~min}$ $(\bullet), 50 \min (\nabla), 180 \min (\diamond), 480 \min (\triangle), 900 \min (+), 1800$ $\min (*)$, and $2880 \mathrm{~min}(\square)$. Wave vectors $k_{0}, k_{1}$, and $k_{c}$ separating the scaling regions are shown for $t=2880 \mathrm{~min}$. The slopes of the dashed lines are $\alpha_{I I I}=0.42$ (for $k_{c}<k<k_{1}$ ), $\alpha_{I I}=0.75$ (for $\left.k_{1}<k<k_{0}\right)$, and $\alpha_{I}=0.99$ (for $\left.k>k_{0}\right)$.

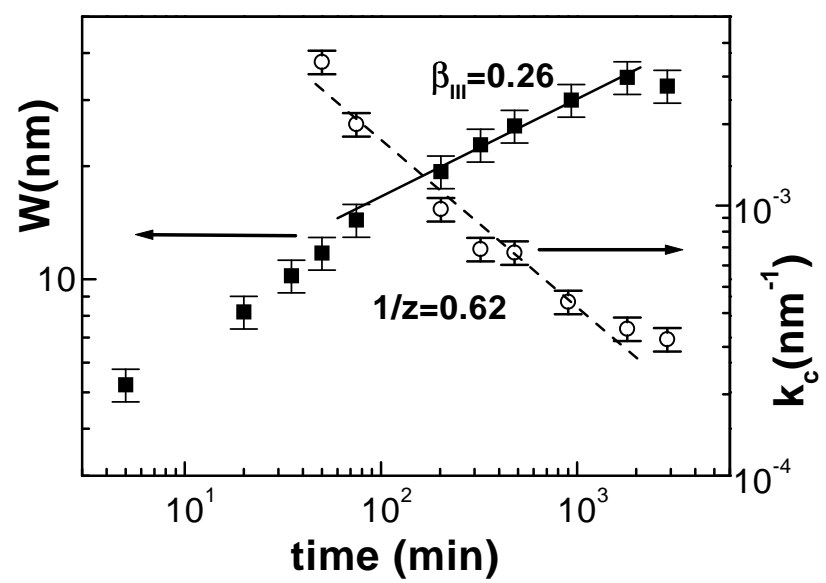

FIG. 3. Plot of $W(t)$ ( $\mathbf{\square}$, left axis) and $k_{c}(t)$ (o, right axis). The slopes of the solid and dashed lines are, respectively, $\beta_{I I I}=0.26$ and $1 / z=0.62$.

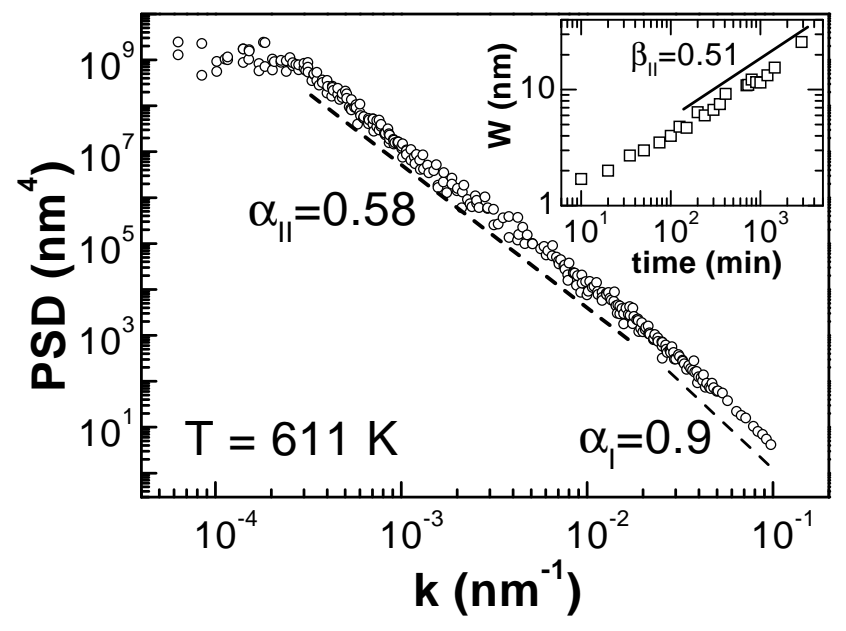

FIG. 4. PSD of a $\mathrm{SiO}_{2}$ film grown for 2 days at $611 \mathrm{~K}$. Slopes of the dashed lines are $\alpha_{I I}=0.58, \alpha_{I}=0.9$. Inset: $W(t)$ for the same system. Slope of the solid line is $\beta_{I I}=0.51$. 


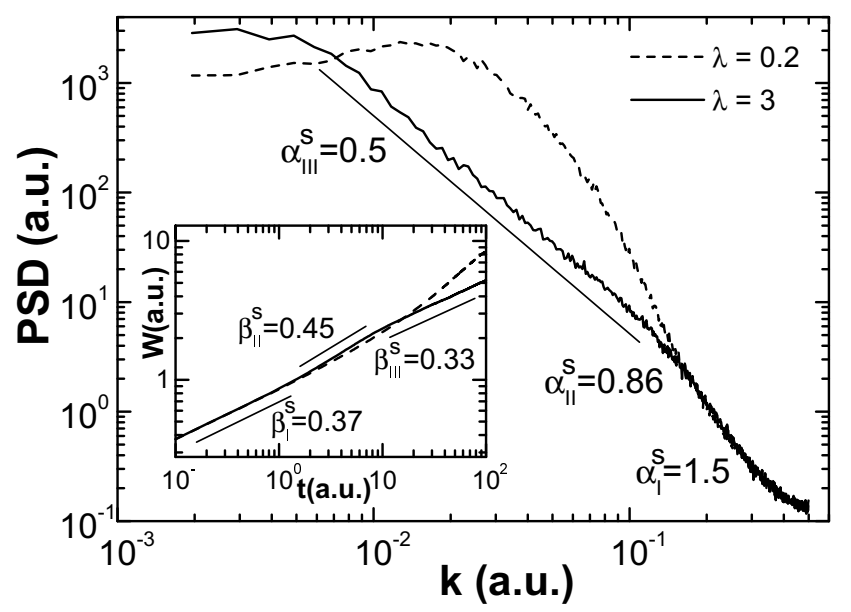

FIG. 5. PSD from numerical integrations of Eq. (2) for $K=D=1, \varepsilon=1 / 2, L=1024, t=100$, averaged over 250 realizations, for $\lambda=0.2$ (dashed line) and $\lambda=3$ (solid line). Slope of the straight solid line is $\alpha_{I I I}^{s}=0.5$. For $\lambda=3$, the values $\alpha_{I}^{s}=1.5, \alpha_{I I}^{s}=0.86$ give the best fit to the PSD behavior in each region. Data curvature at large $k$ is due to discretization effects. Inset: $W(t)$ for the same parameters. Slopes of the straight solid lines are $\beta_{I}^{s}=0.37, \beta_{I I}^{s}=0.45$, and $\beta_{I I I}^{s}=0.33$. 